\title{
Should Open Surgery or Conservative Management be the Choice for Treatment of Ectopic Pregnancy during COVID-19 Pandemic in a Resource-Poor Setting? A Case Series from India.
}

\section{Rahi Pednekar}

Topiwala National Medical College \& BYL Nair Charitable Hospital, Mumbai, India, 400008

Niraj N Mahajan ( $\square$ nirajdr@hotmail.com )

Topiwala National Medical College \& BYL Nair Charitable Hospital, Mumbai, India, 400008

Deepika Tirkey

Topiwala National Medical College \& BYL Nair Charitable Hospital, Mumbai, India, 400008

Geeta Kulkarni

Topiwala National Medical College \& BYL Nair Charitable Hospital, Mumbai, India, 400008 Ankita Pandey

Topiwala National Medical College \& BYL Nair Charitable Hospital, Mumbai, India, 400008

\section{Smita Mahale}

ICMR-National Institute for Research in Reproductive Health, Mumbai, India, 400012

Rahul Gajbhiye ( $\square$ gajbhiyer@nirrh.res.in )

ICMR-National Institute for Research in Reproductive Health, Mumbai, India, 400012

\section{Keywords:}

Posted Date: October 6th, 2020

DOl: https://doi.org/10.21203/rs.3.rs-85956/v1

License: (c) (i) This work is licensed under a Creative Commons Attribution 4.0 International License.

Read Full License 


\section{Abstract}

The authors have requested that this preprint be removed from Research Square. 